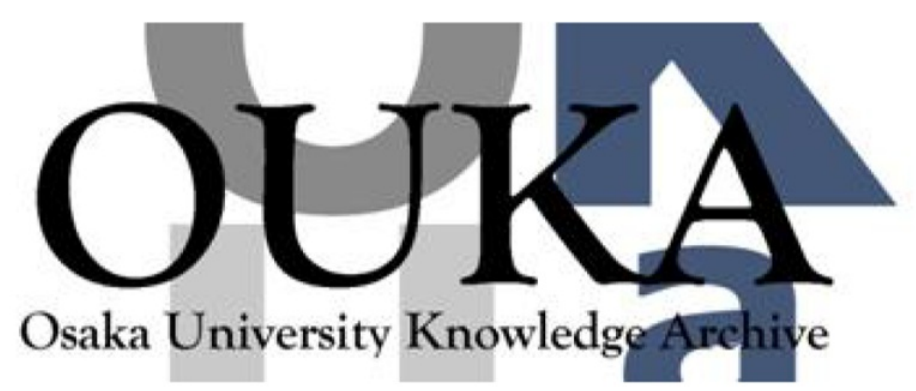

\begin{tabular}{|c|l|}
\hline Title & $\begin{array}{l}\text { Development of shear-vertical-wave point- } \\
\text { focusing electromagnetic acoustic transducer }\end{array}$ \\
\hline Author(s) & $\begin{array}{l}\text { Takishita, Takashi; Ashida, Kazuhiro; Nakamura, } \\
\text { Nobutomo et al. }\end{array}$ \\
\hline Citation & $\begin{array}{l}\text { Japanese Journal of Applied Physics. } 54 \\
\text { p.07HC04 }\end{array}$ \\
\hline Issue Date & $2015-06-05$ \\
\hline oaire:version AM & https://hdl. handle. net/11094/84485 \\
\hline URL & \\
\hline rights & \\
\hline Note & \\
\hline
\end{tabular}

Osaka University Knowledge Archive : OUKA

https://ir. Library. osaka-u. ac. jp/

Osaka University 


\section{Development of shear-vertical-wave point-focusing electromagnetic acoustic transducer}

Takashi Takishita ${ }^{1}$, Kazuhiro Ashida ${ }^{2}$, Nobutomo Nakamura ${ }^{2 *}$, Hirotsugu Ogi ${ }^{2}$, and Masahiko Hirao ${ }^{2}$

${ }^{1}$ School of Engineering Science, Osaka University, Toyonaka, Osaka 560-8531, Japan

${ }^{2}$ Graduate School of Engineering Science, Osaka University, Toyonaka, Osaka 560-8531, Japan

E-mail: nobutomo@me.es.osaka-u.ac.jp

A shear-vertical-wave point-focusing electromagnetic acoustic transducer is developed for the purpose of detecting stress corrosion cracking in stainless steel. The transducer is composed of a permanent magnet and two identical concentric meander-line coils, one for the transmitter and the other for the receiver, and the shear-vertical waves are excited and detected by the Lorentz force mechanism. The meander-line coils are designed so that the phases of all the excited shear-vertical waves are focused in phase at a focal point. The focal area is evaluated experimentally, and it is confirmed that the developed transducer is capable of detecting slit defects deeper than $0.05 \mathrm{~mm}$ at the bottom surface of a stainless-steel plate $20 \mathrm{~mm}$ thick. 


\section{Introduction}

Nondestructive testing is the technique of detecting defects and degradation in materials and structures without destroying them, and the development of advanced transducers is indispensable to ensure the safety of the structures. Stainless steel is widely used, for example, for piping in nuclear energy plants, because of its corrosion resistance. However, welding at a high temperature degrades the corrosion resistance around the welded area, and cracks, so-called stress corrosion cracks (SCCs), appear close to the welded area. ${ }^{1-3)}$ In nuclear energy plants, SSCs lead to the leakage of radioactive materials in the worst case, and they must be detected in the early stage of crack growth. Defects on the inner surface of pipes are detected mainly by two ways: radiographic testing $(\mathrm{RT})^{4,5)}$ and ultrasonic testing (UT). ${ }^{4,6)}$ RT is based on the blackening of radiographic film. It is suitable for detecting defects when their volume is large, but it is not suitable for detecting cracks. On the other hand, UT is suitable for detecting short cracks because acoustic waves show directivity, penetrate into the material, and have a superior reflectance property. For these reasons, UT is often used for detecting SCCs.

For UT, piezoelectric transducers are generally used. ${ }^{7-9)}$ Piezoelectric transducers transmit acoustic waves obliquely from the specimen surface and receive waves reflected by defects. The size and position of the defect are estimated from the amplitude and traveling time of the reflected waves, respectively. Coupling materials are required to transmit acoustic waves from piezoelectric transducers to the specimen. Waveforms obtained with piezoelectric transducers are then easily affected by the amount of coupling material and the surface roughness. The reproducibility is then lowered. In contrast, electromagnetic acoustic transducers (EMATs) ${ }^{10-18)}$ can excite and detect acoustic waves without the need to use coupling materials, leading to higher reproducibility than that of conventional piezoelectric transducers, and they have been used for nondestructive evaluation of weldments. ${ }^{19-21)}$ However, the lower signal transduction efficiency of EMATs than that of piezoelectric transducers has been a disadvantage. A pulsed laser for excitation ${ }^{22,23)}$ and the focusing technique $^{13,24,25)}$ are used to overcome this disadvantage. In this study, we focus on the latter technique and develop the shear vertical (SV)-wave point-focusing EMAT (PF-EMAT).

The fundamental concept of the PF-EMAT is based on the SV-wave line-focusing 
EMAT (LF-EMAT) developed by Ogi et $a l .^{24,25)}$ The LF-EMAT uses the characteristic feature of SV-wave radiation. When the SV wave is excited from a single line source vibrating at the specimen surface parallel to the surface, the radiated wave shows strong directivity; the amplitude of the SV wave propagating at around 30 degree from the normal to the specimen surface is larger than that of others. ${ }^{26)}$ The SV wave is, therefore, suitable for angle beam inspection. In the LF-EMAT, SV waves generated from several line sources are focused on a focal line in phase, and the directivity and spatial resolution are improved. In contrast to the piezoelectric phased array transducer, the focal line of LF-EMAT can be adjusted by changing the pitch of the meander-line coil, and the measurement setup is quite simple. Small defects in an aluminum plate and a steel plate were investigated using conventional LF-EMATs. $^{24,25)}$

In this study, we develop an advanced EMAT to detect slit defects in stainless steel (SUS304), which models SCCs. The transmitting and receiving efficiency of the EMAT is lowered in stainless steel owing to scattering by larger grains, lower electrical conductivity, and higher mass density. However, by achieving the point focusing of SV waves, the efficiency is improved and slit defects deeper than $0.05 \mathrm{~mm}$ are successfully detected in stainless steel.

\section{Directivity of SV wave generated by a single line source}

When a SV wave is generated by a line source vibrating at the surface in the direction normal to the line source and parallel to the surface, the $\mathrm{SV}$ wave shows directivity. ${ }^{26)}$ Figure 1 shows the angle dependence of the radiated amplitude and phase of SV waves in stainless steel, calculated referring to Ref. 26. $\theta$ denotes the radiation angle from the normal to the surface. The amplitude is almost constant between $\theta=0-30^{\circ}$ and shows a sharp peak at about $\theta=32^{\circ}$, beyond which the amplitude drops. The phase remains nearly unchanged up to this angle. The directivity makes the SV wave, especially when excited by a line source such as an EMAT, suitable for detecting defects that exist at a particular position in stainless steel. Strictly speaking, the phase lag must be considered in the design of the EMAT. However, it has been confirmed that the amplitude of the SV wave becomes significantly small for $\theta>$ $32^{\circ}$, and the influence of the phase shift can be neglected. ${ }^{25)}$ In the developed PF-EMAT, which will be described later, SV waves are excited from seven line sources, and two of 
them show phase lags of 1.09 and 1.38 rad. Their relative amplitudes are 0.56 and 0.3 , respectively. The phase lags are less than half of $\pi$, and their amplitudes are not very high. Therefore, the phase lag is not considered in the following development of the PF-EMAT.

\section{SV-wave point-focusing EMAT}

The SV-wave PF-EMAT is composed of a permanent magnet and two concentric meander-line coils for transmitting and receiving SV waves. The coils are located on the surface of a metal specimen, and a permanent magnet is placed on them. The traction forces for SV waves are generated by the Lorentz force mechanism from the interaction of the static magnetic field of the magnet and the eddy currents induced by the coil. ${ }^{27)}$ The receiving coil detects $\mathrm{SV}$ waves from the strain field generated by the SV waves by the same mechanism.

A line-focusing method for the SV wave has been proposed in the literature ${ }^{24)}$, and we extent the principle to point focusing. Figure 2 shows a schematic explanation of the relationship between the focal point and the coil configuration. $\left(0, z_{F}\right)$ is the location of the focal point, $\lambda$ the wavelength of the $\mathrm{SV}$ wave, $r_{i}$ the radius of the $i$ th segment of the coil, and $R_{i}$ the distance between the focal point and the $i$ th segment. Once $z_{F}, \lambda$, and $r_{1}$ are determined, $R_{i}$ is determined so that the difference between the propagation paths of the $i$ th and $(i+1)$ th segments becomes a half-wavelength, $R_{i+1}-R_{i}=\lambda / 2$, in order to make the phases of all the waves equal at the focal point. Then, $r_{i}$ is determined from $R_{i}$ and $z_{F}$.

In this study, slit defects imitating SCCs are fabricated on the bottom surface of a 20-mm-thick stainless-steel (SUS304) plate, which is inspected from the top surface. This means that $z_{F}$ is $20 \mathrm{~mm}$. The PF-EMAT is operated at $2 \mathrm{MHz}$, and the sound velocity of the shear wave is set to be $3100 \mathrm{~m} / \mathrm{s}$ in stainless steel. $\lambda$ is then $1.55 \mathrm{~mm}$. The present PF-EMAT consists of two identical concentric meander-line coils for transmitting and receiving purposes. The diameter of $\mathrm{Cu}$ wires used for the coils is $0.1 \mathrm{~mm}$. The coil possesses seven line sources, and the shape is designed so that radiation angle $\theta$ from each source is between 13.4 and $37.5^{\circ}$. Then, $r_{1}$ and $r_{7}$ are 4.75 and $15.3 \mathrm{~mm}$, respectively. Figure 3 shows the handmade coils. The number of wires of each line source is increased, 4-16, in order to enhance the intensity of the generated SV waves, although the focal area would become larger than the ideal (single line) coils shown in Fig. 2(a). 


\section{Focal area of SV waves generated by the PF-EMAT}

We first measure the focal area of SV waves on the bottom surface. The PF-EMAT is placed on the top surface of the stainless-steel plate, and SV waves are excited by one of the coils. The amplitude distribution of SV waves is measured by scanning the bottom surface with a pinducer (CTS Valpey VP-1093), on which a needle is attached to measure the local-area amplitude. A measurement system with a gated-amplifier and a superheterodyne spectrometer (RITEC RAM-10000) is used for driving the PF-EMAT and receiving the signals detected by the pinducer. High-power RF bursts are applied to the transmitting coil. The driving frequency is $2 \mathrm{MHz}$ and the burst duration is $6 \mu \mathrm{s}$. The pinducer is moved around the focal point every $1 \mathrm{~mm}$. The received waveforms are sent to the superheterodyne spectrometer, and amplitude is calculated. The intended signal is gated out from the waveform, and the amplitude of the driving-frequency component is calculated by the Fourier transformation. The amplitude is then multiplied with the gate width. It is called the time-integrated amplitude hereafter. Figure 4 shows the measured time-integrated amplitude distribution. The designed focal point is located at $(x, y)=(5,5)$, and we observed the maximum amplitude around the intended focal point; the length of the focal area is about $4 \mathrm{~mm}$ in the $x$ and $y$ directions. The measured distribution represents the focal area of the transmitting coil. In the receiving process using the receiving coil, the peak amplitude will be further enhanced because the receiving coil possesses the same focusing effect and only the reflected waves from the focal point are received.

\section{Slit defect detection}

Seven slit defects imitating SCCs are fabricated on the bottom surface of a 20-mm-thick stainless-steel plate, and the slit detectability is investigated. The slit defects have the same width $(0.5 \mathrm{~mm})$ and length $(10 \mathrm{~mm})$, but different depths $(1.45,0.80,0.50,0.45,0.20,0.15$, and $0.05 \mathrm{~mm}$ ). The PF-EMAT is used for both transmitting and receiving, and the same measurement system, driving frequency, and duration time as described above are adopted. The PF-EMAT is placed on the top surface and moved every $1 \mathrm{~mm}$ in the $x$-direction, parallel to a slit defect, as shown in Fig. 5(a). The transmitting coil generates SV waves that are then reflected by the slit defect and received by the receiving coil. The received 
signals are sent to the superheterodyne spectrometer to measure the amplitude of the driving-frequency component.

Figures 5(b)-5(d) show typical waveforms obtained from the flawless area and from the slit defects of 0.05 and $0.15 \mathrm{~mm}$ depth. The signals from the slits clearly appear in the waveforms. On the other hand, no signal was observed when the LF-EMAT was used, and it was confirmed that the slit-defect detectability of PF-EAMT is greater than that of LF-EMAT. Figure 5(e) shows the amplitude profile measured at $x=2-28 \mathrm{~mm}$ for the seven slit defects. Slit defects are located at $x=10-20 \mathrm{~mm}$. The PF-EMAT successfully detects all slit defects. Except for the 1.45-mm-deep slit defect, the amplitude of the reflected signal decreases as the slit depth decreases. A notable feature is the nonlinear depth dependence of the amplitude. For defects of $d=0.15 \mathrm{~mm}$ and deeper, amplitude is not proportional to the defect depth; the depth of the $d=0.15 \mathrm{~mm}$ slit is about $19 \%$ that of the $d=0.80 \mathrm{~mm}$ slit, but the amplitude is about $54 \%$, indicating that the amplitude is not proportional to the slip depth. However, the amplitude suddenly drops at $d=0.05 \mathrm{~mm}$. This drop indicates that the vertical length of the focal area is less than $0.15 \mathrm{~mm}$. Regarding the $d=1.45 \mathrm{~mm}$ slit, the amplitude is lower than that from the $0.80-\mathrm{mm}$-deep defect. The reason for this is unknown at present.

In Fig. 5(e), the amplitude profiles exhibit sharp edges at the ends of the defects, confirming that the focal size of the PF-EMAT is small enough to detect the slit defects. For each slit, the amplitude started to increase at about $x=8 \mathrm{~mm}$, arrived at the peak level at about $x=12 \mathrm{~mm}$, started to decrease at about $x=18 \mathrm{~mm}$, and then arrived at the noise level at $x=22 \mathrm{~mm}$. From these results, the representative length of the focal area of SV waves generated by PF-EMAT is determined to be about $4 \mathrm{~mm}$ in the $x$ direction. This length is close to the size of the focal area observed in Fig. 4.

Finally, we fabricated a 1.50-mm-deep defect with $0.5 \mathrm{~mm}$ width and $10 \mathrm{~mm}$ length on another stainless-steel plate $20 \mathrm{~mm}$ thick, and scanned around it with the PF-EMAT. The amplitude distribution is shown in Fig. 6. The $x$ axis is parallel to the slit defect and the $y$ axis is normal to it. The PF-EMAT is moved in increments of $1 \mathrm{~mm}$ in the $x$ and $y$ directions and the amplitude of the received signal is measured at each point. The slit defect is located at $x=5-15 \mathrm{~mm}$ and $y=5-5.5 \mathrm{~mm}$. The location and shape of the defect are clear in the figure, and this result confirms that the point-focusing of SV waves enables the 
detection of a defect of $0.5 \mathrm{~mm}$ width.

\section{Conclusions}

We developed a PF-EMAT that makes use of the interference of SV waves generated from line sources, and successfully detected slit defects of $0.05 \mathrm{~mm}$ depth at minimum. The size of the focal area was evaluated by measuring the amplitude distribution on the bottom surface, the amplitude profile in the lateral direction of the defect, and also the amplitude distribution around a defect. From these results, the focal size of the PF-EMAT was shown to be small enough to detect the present slit defects.

These results demonstrate the feasibility of detecting defects in stainless steel using the PF-EMAT, and it is expected that the PF-EMAT will replace the conventional piezoelectric transducers. In the future, we will apply the PF-EMAT to detecting SCCs in real stainless-steel components and endeavor to enhance the sensitivity of the PF-EMAT to broaden its range of application. 


\section{References}

1) M. Fox, J. Mater. Eng. Syst. 1, 3 (1979).

2) Y. Ando, Nucl. Eng. Des. 87, 239 (1985).

3) Stress Corrosion Cracking in Light Water Reactors: Good Practices and Lessons Learned (IAEA, Vienna, 2011) p. 19.

4) P. Höller, G. Hübschen, and H. J. Salzburger, Nucl. Eng. Des. 87, 193 (1985).

5) T. Kashiwa, S. Higuchi, and M. Ooka, Mater. Trans. 53, 311 (2009).

6) J. C. Collingwood, NDT Int. 20, 33 (1987).

7) J. Krautkrämer and H. Krautkrämer, Ultrasonic Testing of Materials (Springer, Berlin, 1990) p.117.

8) R. J. Ditchburn, S. K. Burke, and C. M. Scale, NDT\&E Int. 29, 111 (1996).

9) M. Ikeuchi, K. Jinno, Y. Ohara, and K. Yamanaka, Jpn. J. Appl. Phys. 52, 07HC08 (2013).

10) W. D. Wallace, Int. J. Nondestr. Test. 2, 309 (1971).

11) T. J. Moran and R. M. Panos, J. Appl. Phys. 47, 2225 (1976).

12) R. B. Thompson, IEEE Trans. Sonics Ultrason. SU-25, 7 (1978).

13) G. A. Alers and D. T. MacLauchlan, in Review of Progress in Quantitative Nondestructive Evaluation, ed. D.O. Thompson and D. E. Chimenti (Plenum, New York, 1982) Vol. 2A, p. 271.

14) W. B. Maxfield and C. M. Fortunko, Mater. Eval. 41, 1399 (1983).

15) K. Kawashima, IEEE Trans. Sonics Ultrason. SU-32, 514 (1985).

16) R. B. Thompson, in Physical Acoustics, eds. R. N. Thurston and A. D. Pierce (Academic Press, New York, 1990) Vol. 19, p. 157.

17) K. Kawashima and O. B. Wright, J. Appl. Phys. 72, 4830 (1992).

18) Nurmalia, N. Nakamura, H. Ogi, and M. Hirao, Jpn. J. Appl. Phys. 52, 07HC14 (2013).

19) C. M. Fortunko and R. B. Thomson, Proc. ARPR/ARML Review of Progress in Quantitative NDE, 1977-1978, p. 7.

20) C. M. Fortunko and J. C. Moulder, Ultrasonics 20, 113 (1982).

21) K. Sawaragi, H. J. Salzburger, G. Hübschen, K. Enami, A. Kirihigashi, and M. Tachibana, Nucl. Eng. Des. 198, 153 (2000).

22) D. A. Oursler and J. W. Wagner, in Review of Progress in Quantitative Nondestructive Evaluation, ed. D.O. Thompson and D. E. Chimenti (Plenum, New York, 1995) Vol. 14, 
p. 553.

23) S. Dixon, C. Edwards, and S. B. Palmer, Ultrasonics 37, 273 (1999).

24) H. Ogi, M. Hirao, and T. Ohtani, J. Acoust. Soc. Am. 103, 2411 (1998).

25) H. Ogi, M. Hirao, and T. Ohtani, IEEE Trans. Ultrason. Ferroelectr. Freq. Control 46, 341 (1999).

26) G. F. Miller and H. Pursey, Proc. R. Soc. London, Ser. A 223, 521 (1954).

27) H. Ogi, J. Appl. Phys. 82, 3940 (1997). 


\section{Figure Captions}

Fig. 1. Directivity of amplitude and phase of SV waves generated by a single line source at surface of stainless-steel plate vibrating parallel to the surface.

Fig. 2. Configuration of concentric meander-line coils. (a) Top view and (b) cross-sectional view.

Fig. 3. (Color online) Handmade coils. Arrows indicate the current direction.

Fig. 4. (Color online) Amplitude distribution of SV waves generated by the PF-EMAT measured on the bottom surface using the pinducer.

Fig. 5. Experimental results of slit detection. (a) Experimental setup. (b)-(d) Typical waveforms obtained from flawless area and slit defects of 0.05 and $0.15 \mathrm{~mm}$ depth at $x=15$ mm. (e) Amplitude profiles measured at $x=2-28 \mathrm{~mm}$ for the seven slit defects. $d$ denotes the slit depth.

Fig. 6. (Color online) Amplitude distribution around the defect of $1.5 \mathrm{~mm}$ depth, measured by scanning with the PF-EMAT. 


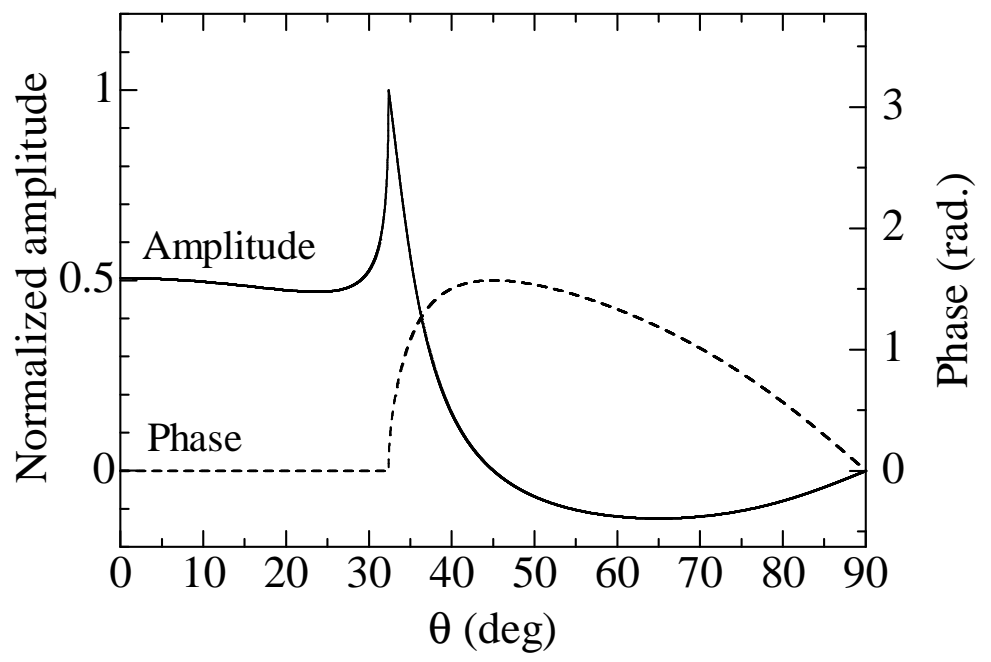

Fig.1. 


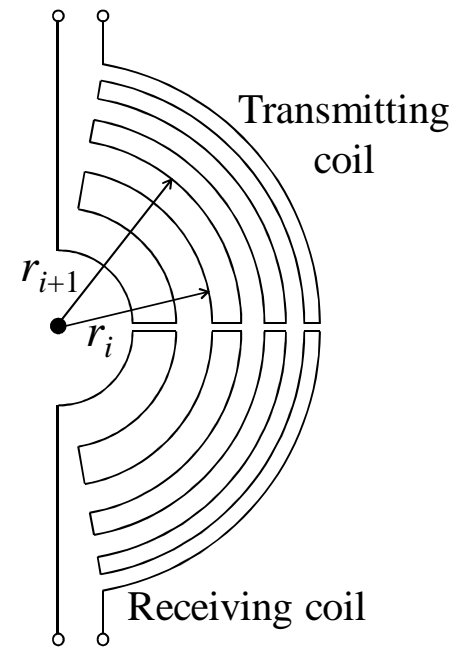

(a)

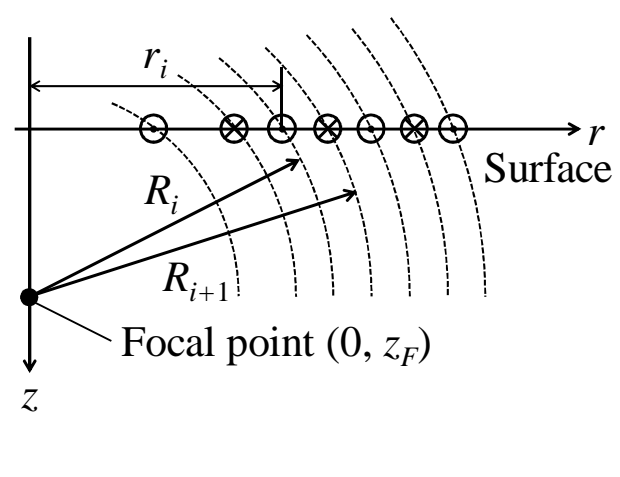

(b)

Fig.2. 


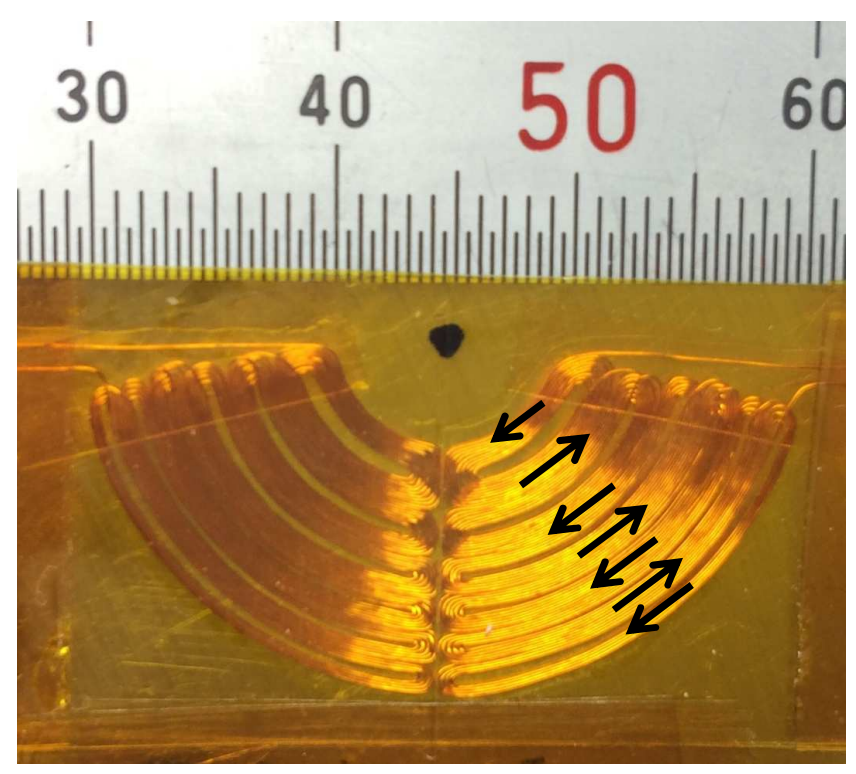

Fig. 3. (Color online) 


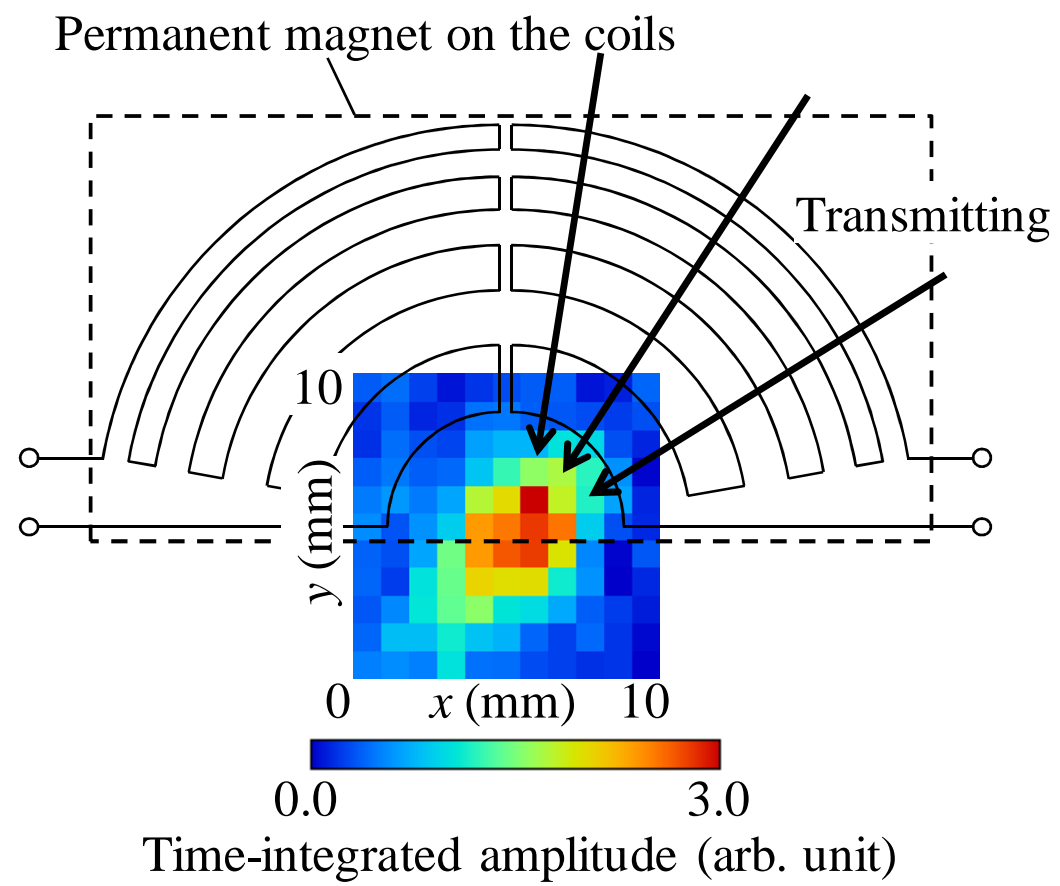

Fig. 4. (Color online) 


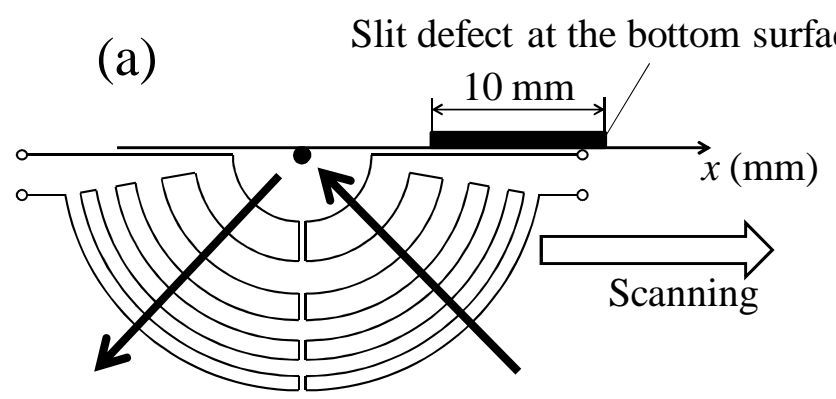

PF-EMAT on the top surface
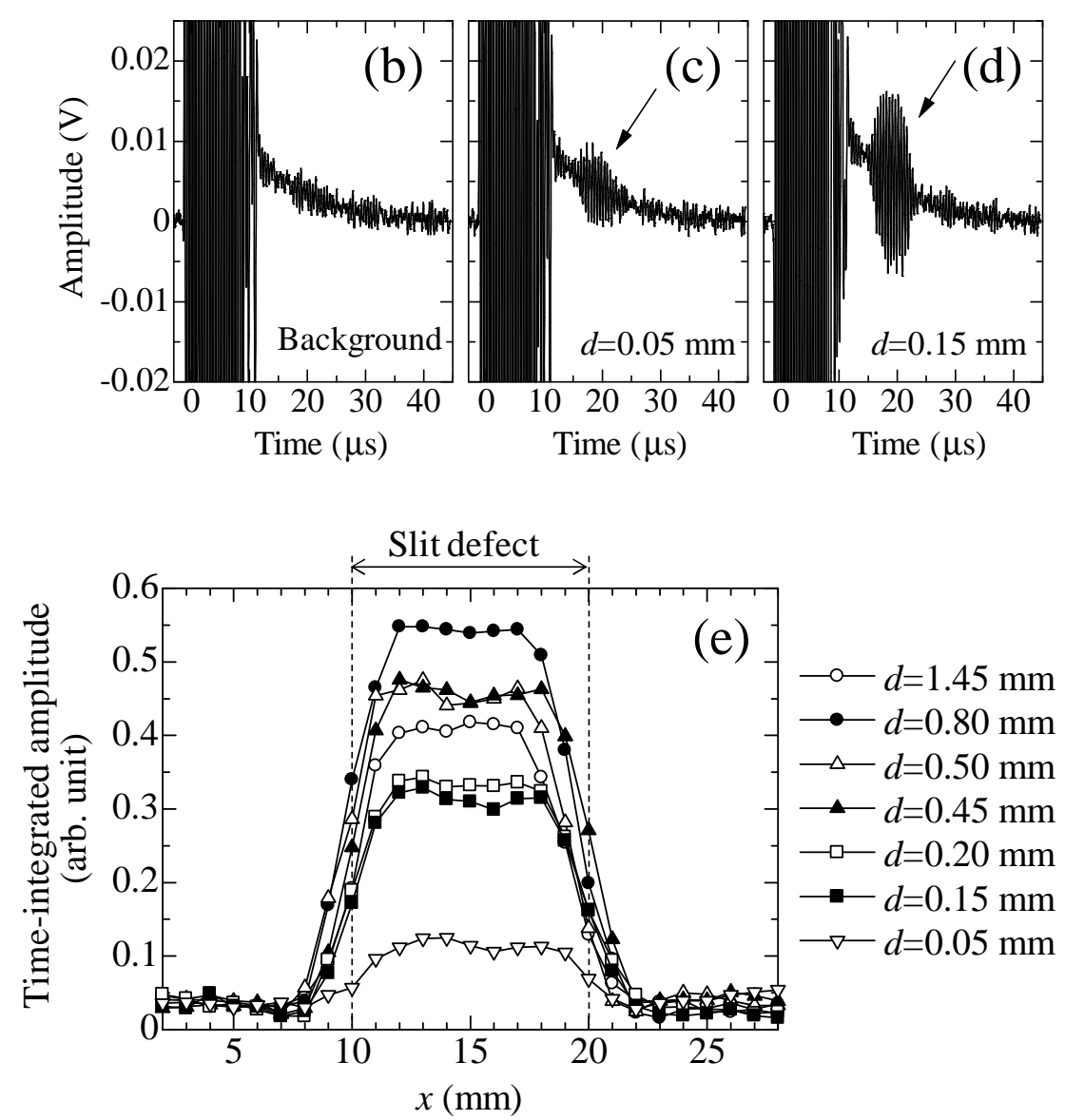

Fig. 5. 
Slit defect at the bottom surface
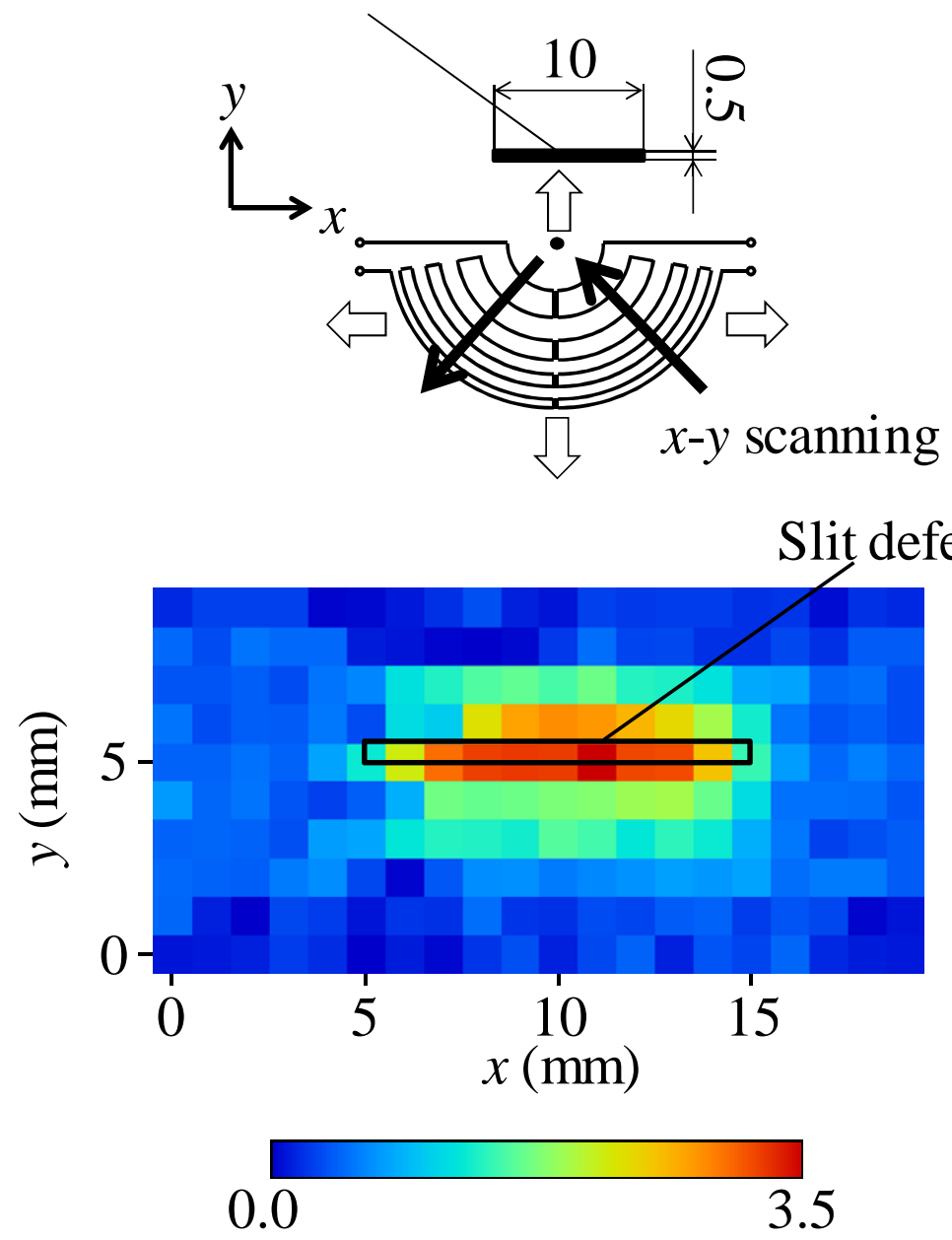

Time-integrated amplitude (arb. unit)

Fig. 6. (Color online) 